\title{
One-dimensional bipolaron in the strong-coupling limit
}

\author{
P. Vansant* and F. M. Peeters ${ }^{\dagger}$ \\ Universiteit Antwerpen (UIA), Departement Natuurkunde, Universiteitsplein 1, B-2610 Antwerpen, Belgium \\ M. A. Smondyrev ${ }^{\ddagger}$ and J. T. Devreese $e^{\S}$ \\ Technische Universteit Eindhoven, Departement Natuurkunde, P.O. Box 513, 5600 MB Eindhoven, The Netherlands
}

(Received 31 January 1994; revised manuscript received 6 July 1994)

\begin{abstract}
The one-dimensional (1D) large bipolaron is investigated in the limit of strong electron-phonon coupling. The nonlinear integro-differential equation for the bipolaron wave function is solved numerically, from which we obtained estimates for the main characteristics. An enlargement of the stability region for the bipolaron ground state is found in $1 \mathrm{D}$ as compared to the stability regions in $2 \mathrm{D}$ and $3 \mathrm{D}$. The energy of the first relaxed excited state (RES) equals the energy of two single polarons and the ground state in the potential generated by the first RES has a slightly lower energy than this RES and is therefore stable. The nonlinearity causes the feature that the combination of the ground state and an excited state of one-particle wave functions could lead to a higher bipolaron energy than the combination of two excited states.
\end{abstract}

\section{INTRODUCTION}

Two electrons in a polar or ionic crystal will interact with each other through (1) the direct repulsive Coulomb interaction and (2) an attractive interaction caused by the polarization of the surrounding lattice. If the Coulomb interaction is sufficiently screened by the lattice and if the polaron ${ }^{1}$ interaction is sufficiently strong, a bound state between the two electrons and the surrounding common cloud of virtual phonons may be formed. Such a composite quasiparticle is referred to as a bipolaron.

Using Coulombic type of wave functions for each electron, Vinetskii, ${ }^{2}$ Mukhomorov, ${ }^{3}$ Suprun and Moizhes ${ }^{4}$ obtained rather wide stability regions for the bipolaron in the limit of an infinitely large electron-phonon coupling constant $\alpha$. Adamowski ${ }^{5}$ and later Bassani et al. ${ }^{6}$ used variational calculations but compared the bipolaron ground state energy with more correct single-polaron energies. Verbist et al. ${ }^{7,8}$ used a Feynman path-integral technique in combination with a conventional variational approach and obtained more accurate results, especially in two dimensions (2D).

The study of bipolarons can be relevant to examine the applicability of the bipolaron theory to high- $T_{c}$ superconductivity (e.g., for high- $T_{c}$ superconductors, ${ }^{9,10}$ the recent discovered fullerites ${ }^{11,12}$ and the proposed BoseEinstein condensation of large bipolarons ${ }^{13,14}$ ).

It is interesting also to study relatively simple systems such as $1 \mathrm{D}$ bipolarons where charges are confined to a one-dimensional structure, which could be relevant in quantum wires ${ }^{15}$ and linear conjugated organic polymer conductors. ${ }^{16}$ In Ref. 17 it was recently proved that in the limit of a very strong magnetic field the 3D bipolaron maps into a $1 \mathrm{D}$ bipolaron problem.

The aim of the paper is to present a more accurate and detailed treatment of 1D bipolaron formation in the strong-coupling limit. In this limit the adiabatic approximation leads to exact equations because the electron os- cillations have a much higher frequency than the lattice frequency $\omega_{L O}$. Besides a variational method we use also a Fourier series expansion of the wave function. This expansion leads in principle to an exact solution. We will consider the main characteristics (e.g., the energy, the stability region, etc.) of one $1 \mathrm{D}$ bipolaron.

With the Fourier series approach we are also able to investigate the excited states of the $1 \mathrm{D}$ bipolaron. The relaxed excited states (RES's) and Franck-Condon (FC) excited states of single polarons in $3 \mathrm{D}$ were studied by Devreese and co-workers. ${ }^{18-20}$ For their role on the surface of a liquid-helium film see Ref. 21.

The paper is organized as follows. In Sec. II we introduce the nonlinear Schrödinger equation for the 1D bipolaron. This integro-differential equation is solved variationally in Sec. III and by a Fourier series expansion in Sec. IV. Also a comparison is made between estimates for bipolaron charateristics calculated within the two approaches. In Sec. V we investigate the excited states of the $1 \mathrm{D}$ bipolaron and in Sec. VI the conclusions are given.

\section{STRONG-COUPLING LIMIT}

The one-dimensional bipolaron Hamiltonian, which is the Hamiltonian of two electrons interacting with a phonon field, is given by

$$
\begin{aligned}
H= & \sum_{j=1,2} \frac{p_{j}^{2}}{2 m}+U\left(z_{1}-z_{2}\right)+\sum_{k} \hbar \omega_{k} b_{k}^{\dagger} b_{k} \\
& +\sum_{j=1,2} \sum_{k}\left[V_{k} b_{k} e^{i k z_{j}}+V_{k}^{*} b_{k}^{\dagger} e^{-i k z_{j}}\right]
\end{aligned}
$$

where $z_{j}$ and $p_{j}$ are the position and momentum operators of the $j$ th electron $(j=1,2), m$ is the electron band mass, and $\omega_{k}$ is the frequency of the phonons with wave vector $k$. 
In the case of an electron interacting with the LO mode we have dispersionless phonons $\omega_{k}=\omega_{L O}$ and the interaction coefficients are

$$
V_{k}=-i \hbar \omega_{L O} \sqrt{\frac{2 \alpha^{\prime}}{L} \sqrt{\frac{\hbar}{2 m \omega_{L O}}}}
$$

with $L$ the length of the system and $\alpha^{\prime}$ the coupling constant for the one-dimensional electron-phonon interaction (defined in Refs. 22 and 23): $\alpha^{\prime}=$ $\alpha \sqrt{\pi} \Gamma((D-1) / 2) / 2 \Gamma(D / 2)$. Here $\alpha$ is the standard dimensionless electron-phonon coupling constant in $3 \mathrm{D}$ and $D$ the number of space dimensions. For $D \rightarrow 1$ we have $\alpha^{\prime}=\alpha /(D-1)$. Indeed, the polaron characteristics diverge for $D=1$ because of the Coulombic nature of this problem. By using the renormalized coupling constant $\alpha^{\prime}$ rather than $\alpha$, all expressions are regularized and finite results are found for the energy and the mass of a polaron or bipolaron in $1 \mathrm{D}$.

The 3D Coulomb repulsion between the electrons is defined by

$$
\begin{aligned}
\frac{U}{r} & =U_{D}^{\prime} \frac{\Gamma(D / 2)}{\pi^{D / 2+1}} \int \frac{d^{D} \mathbf{k}}{k^{D-1}} \exp [i \mathbf{k r}] \\
U_{D}^{\prime} & =U \sqrt{\pi} \frac{\Gamma((D-1) / 2)}{2 \Gamma(D / 2)}
\end{aligned}
$$

with $D=3$. In the limit $D \rightarrow 1$ the corresponding Coulomb potential takes the form of the so-called contact potential $2 U_{1}^{\prime} \delta\left(z_{1}-z_{2}\right)$. Therefore we have in (2.1) $U\left(z_{1}-\right.$ $\left.z_{2}\right)=2 U_{1}^{\prime} \delta\left(z_{1}-z_{2}\right)$. It is convenient to introduce also the dimensionless coupling constant $U^{\prime}$,

$$
U_{1}^{\prime}=\hbar \omega_{L O} \sqrt{\frac{\hbar}{m \omega_{L O}}} U^{\prime}
$$

and the ratio of the coupling constants,

$$
g=\frac{U^{\prime}}{\alpha^{\prime}}=\frac{\sqrt{2}}{1-\eta}
$$

where $\eta=\epsilon_{\infty} / \epsilon_{0}$ with $\epsilon_{\infty}\left(\epsilon_{0}\right)$ the high frequency (static) dielectric constant.

Starting from the Hamiltonian (2.1) and using the adiabatic approximation (the ions are at rest) we arrive at the nonlinear effective Schrödinger equation for the onedimensional bipolaron in the strong-coupling limit (for more details see Refs. 24 and 25). Using the symmetry of the wave function $\left|\chi\left(z_{1}, z_{2}\right)\right|^{2}=\left|\chi\left(z_{2}, z_{1}\right)\right|^{2}$ which allows symmetrical as well as antisymmetrical solutions, we obtain

$\left\{-\frac{1}{2} \frac{\partial^{2}}{\partial z_{1}^{2}}-\frac{1}{2} \frac{\partial^{2}}{\partial z_{2}^{2}}+\tilde{U}\left(z_{1}, z_{2} ; \chi\right)\right\} \chi\left(z_{1}, z_{2}\right)=\varepsilon \chi\left(z_{1}, z_{2}\right)$

with

$$
\begin{aligned}
\tilde{U}\left(z_{1}, z_{2} ; \chi\right)= & 4 \sqrt{2} \int_{-\infty}^{\infty} d x_{1} d x_{2} d x_{2}^{\prime} \chi^{2}\left(x_{1}, x_{2}\right) \chi^{2}\left(x_{1}, x_{2}^{\prime}\right) \\
& -4 \sqrt{2} \int_{-\infty}^{\infty} d x_{2}^{\prime}\left[\chi^{2}\left(z_{1}, x_{2}^{\prime}\right)+\chi^{2}\left(z_{2}, x_{2}^{\prime}\right)\right] \\
& +2 g \delta\left(z_{1}-z_{2}\right)
\end{aligned}
$$

and with $\varepsilon=E_{0} / \hbar \omega_{L O} \alpha^{\prime 2}$. To obtain Eq. (2.6) a scaling is performed which made all the quantities in it, e.g., the positions $z_{i}$, the energy $\varepsilon$, and the wave functions, dimensionless.

\section{VARIATIONAL APPROACH}

By multiplying both sides of (2.6) with $\chi\left(z_{1}, z_{2}\right)$ and after integration over $z_{1}$ and $z_{2}$ the above integrodifferential equation (2.6) can be rewritten as a variational problem. The ground state energy can then be obtained from the minimization of the obtained functional with respect to $\chi\left(z_{1}, z_{2}\right)$. However, it is convenient to introduce the center-of-mass and relative coordinates

$$
\begin{array}{ll}
Z=\frac{z_{1}+z_{2}}{2}, & P=p_{1}+p_{2}, \\
z=z_{1}-z_{2}, & p=\frac{p_{1}-p_{2}}{2} .
\end{array}
$$

We use the notation $\chi\left(z_{1}, z_{2}\right)=\Psi(Z, z)$ and the symmetry $\left|\chi\left(z_{1}, z_{2}\right)\right|^{2}=\left|\chi\left(z_{2}, z_{1}\right)\right|^{2}$ of the wave function. The functional then takes the form

$$
\begin{aligned}
A[\Psi]= & \frac{1}{4} \int_{-\infty}^{\infty} d Z d z\left(\frac{\partial \Psi(Z, z)}{\partial Z}\right)^{2} \\
& +\int_{-\infty}^{\infty} d Z d z\left(\frac{\partial \Psi(Z, z)}{\partial z}\right)^{2} \\
& -4 \sqrt{2} \int_{-\infty}^{\infty} d Z d z d z^{\prime} \Psi^{2}(Z, z) \Psi^{2}\left(Z+z / 2, z^{\prime}\right) \\
& +2 g \int_{-\infty}^{\infty} d Z \Psi^{2}(Z, 0) .
\end{aligned}
$$

Following Ref. 8 we consider the product ansatz

$$
\Psi(Z, z)=\Phi(Z) \phi(z)
$$

and choose a Gaussian trial wave function for the centerof-mass motion,

$$
\Phi(Z)=\left[\frac{\omega}{2 \pi}\right]^{1 / 4} \exp \left(-\frac{\omega Z^{2}}{4}\right)
$$

which leads to the functional

$$
\begin{aligned}
A[\phi]= & \frac{\omega}{16}+\omega \int_{-\infty}^{\infty}\left(\frac{d \phi}{d z}\right)^{2} d z+2 \sqrt{2 \omega}\left[\frac{\phi^{2}(0)}{1-\eta}-\frac{1}{\pi}\right. \\
& \left.\times \int_{-\infty}^{\infty} d z_{1} d z_{2} \exp \left(-\frac{\left(z_{1}+z_{2}\right)^{2}}{16}\right) \phi^{2}\left(z_{1}\right) \phi^{2}\left(z_{2}\right)\right]
\end{aligned}
$$

with $\omega$ a variational parameter. The ground state bipolaron energy $E_{\text {bip }}$ (in units of $\hbar \omega_{L O} \alpha^{\prime 2}$ ) is then to be 
obtained by minimizing the functional $A[\phi]$ with respect to $\omega$ and $\phi$.

The bipolaron can exist as a stable state when the bipolaron energy $E_{\text {bip }}$ is lower than twice the single polaron energy $E_{\text {pol }}$. In the strong-coupling limit $E_{\text {bip }} \sim$ $\alpha^{\prime 2}$ and $E_{\mathrm{pol}} \sim \alpha^{\prime 2}$, and as a consequence $\alpha^{\prime 2}$ can be factored out. The bipolaron formation is then exclusively determined by the physical parameter $\eta=\varepsilon_{\infty} / \varepsilon_{0}$. It is found that a critical value $\eta_{c}$ exists below which a bipolaron state will be energetically more favorable than a state with two single polarons.

Gross ${ }^{26}$ showed that the analogous strong-coupling 1D polaron problem could be solved exactly in the adiabatic approximation (see also Ref. 27) from which we obtain the exact polaron energy in dimensionless units [cf. definition of $\varepsilon$ in Eq. (2.6)]: $\varepsilon_{\text {pol }}^{\text {exact }}=-1 / 3$. When a Gaussian trial wave function is $\operatorname{chosen}^{23}$ the energy is $\varepsilon_{\text {pol }}^{\text {Gauss }}=-1 / \pi$.

Of course, the condition for bipolaron formation will depend on the value of $\varepsilon_{\text {pol }}\left(\varepsilon_{\text {pol }}^{\text {exact }}\right.$ or $\left.\varepsilon_{\text {pol }}^{\text {Gauss }}\right)$ we choose (see, e.g., Ref. 8). The corresponding $\eta_{c}$ will be denoted by $\eta_{c}^{\text {low }}$ for $\varepsilon_{\text {pol }}=\varepsilon_{\text {pol }}^{\text {exact }}=-1 / 3$ and $\eta_{c}^{\text {approx }}$ for $\varepsilon_{\text {pol }}=\varepsilon_{\text {pol }}^{\text {Gauss }}=-1 / \pi$. The $\eta_{c}^{\text {low }}$ is a lower bound to $\eta_{c}$, due to the fact that the obtained bipolaron energy is an upper bound to the exact energy. As for $\eta_{c}^{\text {approx }}$, it provides us neither an upper nor a lower bound, but as an estimate it could be closer to the exact value than $\eta_{c}^{\text {low }}$. Both $\eta_{c}$ values are considered in this paper. Normally, one should compare the bipolaron energy with twice the energy of the single polaron obtained within the same approximation. However, because the exact single polaron energy $\varepsilon_{\text {pol }}^{\text {exact }}$ is available, we can obtain a lower bound to $\eta_{c}$.

The dependence of the wave function on the relative coordinates is contained in $\phi(z)$. We checked various trial wave functions (for details see Ref. 25). The best result (i.e., the lowest upper bound for the bipolaron ground state energy) was obtained with the function

$$
\phi(z)=N\left(1+B|z|+C z^{2}\right) \exp \left(-\frac{\nu^{2} z^{2}}{4}\right)
$$

where $N$ is the normalization constant and $B, C$, and $\nu$ are variational parameters. In contrast to the $2 \mathrm{D}$ and $3 \mathrm{D}$ cases, the direct repulsion in $1 \mathrm{D}$ is a contact potential, which implies that for the 1D bipolaron ground state: (a) The wave function $\phi(z)$ is not zero for $z=0$, and (b) the first derivative of $\phi(z)$ can be discontinuous at $z=0$. Therefore, the constant term in the polynomial part of $\phi(z)$ is not negligible as it was in 2D and 3D (see Ref. 8). From trying also other trial functions we observe that the term $B|z|$ in the wave function $\phi(z)$ turns out to be very important while the term $C z^{2}$ plays no significant role. Besides, we have found that the exponentially decreasing Coulombic type of functions give smaller binding energies than the Gaussian function (3.6). The opposite is true for the single polaron case. ${ }^{23}$

In Fig. 1 the bipolaron ground state energy, in dimen-

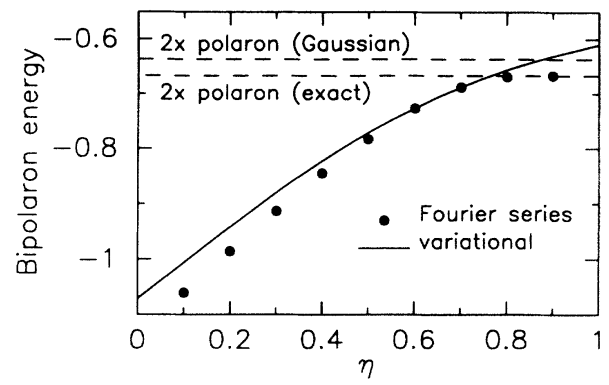

FIG. 1. The 1D bipolaron energy (in dimensionless units) is plotted vs $\eta$ for the best variational trial function (considered here) and for the solution of the integro-differential equation using the Fourier series approach after extrapolation to the $n \rightarrow \infty$ limit $\varepsilon_{F S}^{\infty}$ (points). The horizontal lines give the energy of two separate polarons $\varepsilon=2 \varepsilon_{\mathrm{pol}}$ where (1) $\varepsilon_{\mathrm{pol}}$ is calculated within the Gaussian approach, and (2) $\varepsilon_{\text {pol }}$ is the exact result. The crossings of the curves with the straight lines determine the $\eta_{c}$ values.

sionless units, is plotted as a function of $\eta$ for the above trial function (3.6). The results for this energy could be fitted to the curve $\varepsilon=-1.07+0.737 \eta-0.273 \eta^{2}$, within $1.5 \%$ over the range $\eta \in[0,0.9]$. The two dashed horizontal lines in Fig. 1 correspond to twice the singlepolaron energy calculated using the asymptotical exact wave function and a Gaussian, respectively. The crossings of the ground state bipolaron energy curves with those straight lines determine the critical $\eta_{c}$ value at which bipolaron formation occurs. The trial wave function (3.6) gives the lowest bipolaron ground state energy of all tested trial functions and leads consequently to the largest $\eta_{c}$ value. Because the $\eta_{c}^{\text {low }}$ are lower bounds to $\eta_{c}$, this largest $\eta_{c}^{\text {low }}$ is the best lower bound for $\eta_{c}$ we obtained within the variational approach. We obtain $\eta_{c}^{\text {approx }}=0.874$ and $\eta_{c}^{\text {low }}=0.764$.

\section{FOURIER SERIES APPROACH}

The power of the variational approach lies in the fact that the method provides an upper bound to the bipolaron ground-state energy. This gives a criterium to judge which is the best bipolaron wave function. On the other hand, it is less clear how to improve in a systematic way a given variational wave function. E.g., the presence of the quadratic term in the polynomial factor of the trial function (3.6) does not lower substantially the bipolaron energy (less than $0.4 \%$ ) which might indicate that the resulting bipolaron wave function (3.6) is close to the exact result. Because this is not guaranteed, we present an approach which inherently allows to improve systematically the bipolaron binding energy. Another advantage is that we do not have to make the product ansatz approximation (3.3).

In order to solve (2.6) numerically we put our system in a square box with dimension $[-L, L]$ and infinitely high 
wells. The bipolaron wave function can now be represented as a linear combination of the basis functions of this box,

$$
\chi\left(z_{1}, z_{2}\right)=\sum_{n, m}^{N, M} a_{n m} \sin \frac{n \pi\left(z_{1}+L\right)}{2 L} \sin \frac{m \pi\left(z_{2}+L\right)}{2 L}
$$

Multiply both sides of Eq. (2.6) with $\chi\left(z_{1}, z_{2}\right)$ and integrate over $z_{1}$ and $z_{2}$. The resulting equation constitutes an eigenvalue problem which provides the bipolaron energy and the parameters $a_{n m}$ for a given potential $\tilde{U}$. Because this potential $\tilde{U}$ depends on the wave function $\chi$ in a nonlinear way, we solve the problem iteratively by inserting the found wave function into $\tilde{U}$ until convergence is obtained. In principle this is an exact procedure when $N=M \rightarrow \infty$. In practice, we have to limit ourselves to a finite number of terms in Eq. (4.1), typically $N=M=15$.

The resulting $\eta_{c}^{\text {low }}$ value for $N=M=15$ is 0.672 . Using the symmetry of the ground state we had the possibility to calculate the ground state bipolaron energies $\varepsilon_{F S}$ for some discrete values of $\eta$ with $N=M=30$.

This method gives higher ground state energies, and thus lower binding energies, as compared to the best variational solution. Therefore we investigate the dependence of the bipolaron energy on the number of Fourier terms in our expansion (4.1). We found that the numerical data could be well presented by the curve $\varepsilon_{\text {bip }}=\varepsilon_{0}-B / n$ with $\varepsilon_{0}=-0.783$ and $B=-0.965$ for $\eta=0.5$. This is a very slowly converging series with $n$ which explains why we found less accurate binding energies as compared to the variational approach. Therefore we used this fit $\varepsilon_{\text {bip }}=\varepsilon_{0}-B / n$ in order to obtain the $n=N=M \rightarrow \infty$ results of the Fourier expansion which are also indicated in Fig. 1 by points which we were able to fit to the polynomial: $\varepsilon=-1.176+1.062 \eta-0.551 \eta^{2}$ with an accuracy of $4 \%$ in the region $\eta \in[0,0.9]$. We see that these energies $\varepsilon_{F S}^{\infty}$ are lower than the variational results and tend asymptotically to the energy of two single polarons. The proof of the latter statement is given in the next section. The corresponding $\eta_{c}^{\text {low }}$ is in this case 1 . Therefore, the ground state is always stable and exists.

In Fig. 2 the wave function $\chi\left(z_{1}, z_{2}\right)$ together with its contourplot is shown for $\eta=0.2$ and $N=M=15$. From this figure it is clear that the two electrons avoid each other and that they have the highest probability to be at a well-defined distance from each other.

The critical (maximal) value of the repulsion $U_{c}^{\prime}\left(\alpha^{\prime}\right)$ at a given value of $\alpha^{\prime}$ is determined by the condition $E^{\text {bip }}\left(\alpha^{\prime}, U_{c}^{\prime}\right)=2 E^{\text {pol }}\left(\alpha^{\prime}\right)$ that the bipolaron ground state energy equals the ground state energy of two independent free polarons. The point where the curve $U_{c}^{\prime}\left(\alpha^{\prime}\right)$ goes out of the physical region gives us the critical (minimal) value $\alpha_{c}^{\prime}$ of the electron-phonon coupling constant: $U_{c}^{\prime}\left(\alpha_{c}^{\prime}\right)=\sqrt{2} \alpha_{c}^{\prime}$. Using the Feynman path-integral technique this value was found to be $\alpha_{c}=6.8$ for the $3 \mathrm{D}$ case. $^{7}$ It was proven that within this path-integral approach the polaron ${ }^{22,28,29}$ and bipolaron ${ }^{7}$ ground state energies satisfy scaling relations such that the corre-

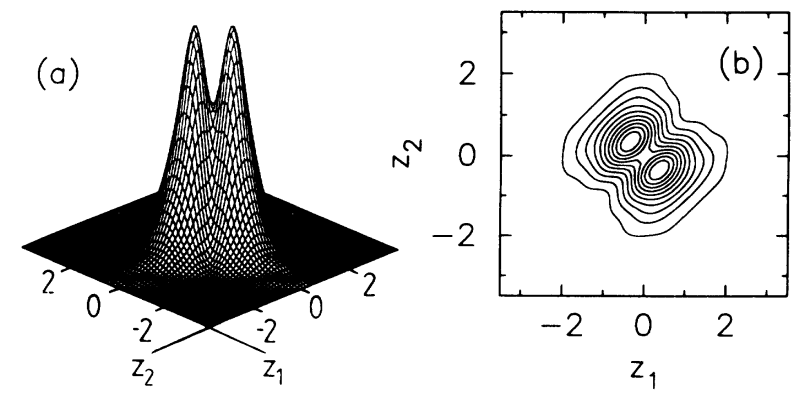

FIG. 2. (a) The 1D bipolaron wave function $\phi\left(z_{1}, z_{2}\right)$ for $\eta=0.2$ as obtained within the Fourier series approach. (b) The contour map corresponding to this bipolaron wave function.

sponding energies in $n \mathrm{D}$ can be obtained from the one in 3D. Applying these scaling relations one obtains ${ }^{17}$ $\alpha_{c}^{\prime}=2.3$ in $1 \mathrm{D}$.

The rms separation (scaled by $\sqrt{\hbar / m \omega_{L O} \alpha^{\prime 2}}$ into dimensionless units) between the electrons is defined by

$$
R=\left[\left\langle z^{2}\right\rangle\right]^{1 / 2}
$$
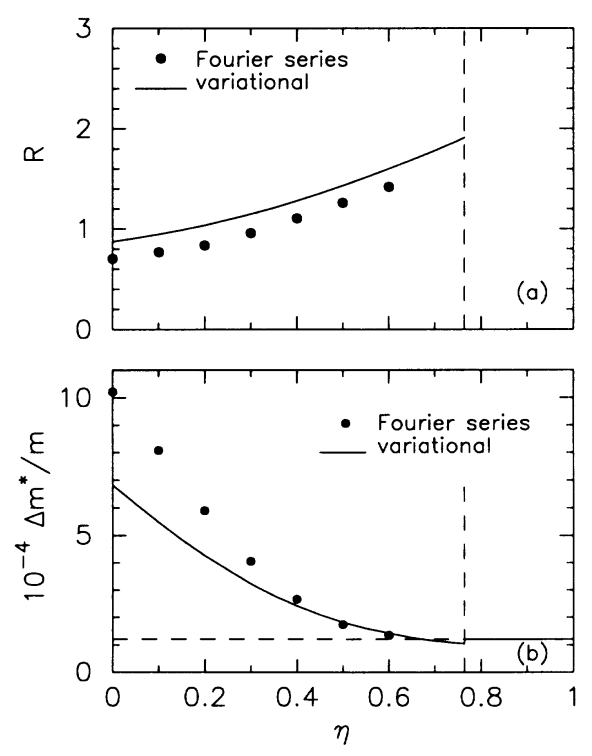

FIG. 3. (a) The rms separation $R$ of the electrons in the bipolaron as a function of $\eta$ is shown for the best (considered here) variational trial function (solid line) and for the wave function obtained by the Fourier series approach (points). The results are only shown when the bipolaron is stable within the corresponding approach $\left(\eta<\eta_{c}^{\text {low }}\right)$. (b) The electron-phonon correction to the effective bipolaron mass for $\alpha^{\prime}=8$ as obtained with the best (considered here) variational trial function (solid line) and the Fourier series method (points). The horizontal line indicates the value of two times the effective mass of a strongly coupled $1 \mathrm{D}$ polaron. 
where the averaging is performed over the wave function. The evolution of $R$ as a function of $\eta$ is shown in Fig. $3(\mathrm{a})$. Notice that $R$ increases smoothly with increasing $\eta$, as expected. For the trial function (3.6) we found for the endpoints of the bipolaron region: $R(\eta=0)=0.88$ and $R\left(\eta=\eta_{c}^{\text {low }}=0.764\right)=1.91$. The rms separation $R$ is also shown in Fig. 3(a) for the Fourier series approach (points). Notice that the Fourier series approach predicts that the electrons are on average closer to each other as compared to the best variational approach. The Fourier series result (extrapolation from $N=M \leq 15$; see later) is only shown when the bipolaron is stable within this approach $\left(\eta \leq \eta_{c}^{\text {low }}=0.672\right)$.

Within the strong-coupling limit the effective bipolaron mass is given by (for details see Ref. 24)

$$
\begin{aligned}
\frac{m^{*}}{m}=2 & +8 \sqrt{2} \alpha^{4} \int_{-\infty}^{\infty} d z_{1} d z_{2} d z_{2}^{\prime}\left[\frac{\partial}{\partial z_{1}} \chi^{2}\left(z_{1}, z_{2}\right)\right] \\
& \times\left[\frac{\partial}{\partial z_{1}} \chi^{2}\left(z_{1}, z_{2}^{\prime}\right)\right]
\end{aligned}
$$

In Fig. 3(b) the result of the electron-phonon correction to the bipolaron mass $\Delta m^{*} / m=m^{*} / m-2$ is shown for the best variational wave function and for the wave function obtained by the Fourier series method. We observe that the variational approach (solid line) reproduces in the limit of $\eta \approx \eta_{c}^{\text {low }}$ (where $\eta_{c}^{\text {low }}$ is obtained within the same variational method) almost the correct asymptotical effective mass of two separated $1 \mathrm{D}$ polarons (dashed line). This asymptotical value is just twice the value obtained by scaling the $3 \mathrm{D}$ effective polaron mass (calculated within the first two orders of the strongcoupling expansion ${ }^{23}$ ). The reason for this choice is that the center-of-mass motion of the bipolaron is treated in a Gaussian approximation [cf. Eq. (3.4)] and thus calculated in the same approximation as the $3 \mathrm{D}$ effective polaron mass.

Also the effective bipolaron mass calculated with the Fourier series method (points) is given for some values of $\eta$ below $\eta_{c}^{\text {low }}=0.672$ obtained with the same method. The Fourier series approach is, however, not accurate enough to obtain good estimates for the effective mass and rms separation (an accurate result for the energy can be extrapolated, but for the wave function with which the effective mass and rms separation are calculated we cannot do this). We used in the calculations of the rms separation and the effective mass the Fourier series approach with $N=M \leq 15$ and extrapolated the results in the limit $N=M \rightarrow \infty$.

\section{EXCITED STATES OF THE 1D BIPOLARON}

With the Fourier series approach we are also able to investigate the excited states of the $1 \mathrm{D}$ bipolaron. Following the definitions given by Devreese ${ }^{18}$ we distinguish three different types of excited states. First of all, there are the ground state and relaxed excited states (RES's). The polarization of the medium is then adapted to the final electronic configuration. These states are described by self-consistent solutions of the nonlinear Schrödinger equation. Then, there are the Franck-Condon (FC) excited states. The electron is then excited while the polarization is remaining at the initial ground state electronic configuration. Finally, there exist also Franck-Condon type excited states which are states available for an electron in a potential well created by one of the RES's.

In what follows we use the quantum number $n \geq 1$ to enumerate the self-consistent solutions and $n^{\prime} \geq 1$ to enumerate the excited states in an effective potential well generated by one of the self-consistent solutions. Therefore, $\varepsilon_{n, n^{\prime}}$ will be the energy of the $n^{\prime}$ th state in the effective potential generated by the $n$th self-consistent solution. It is clear that dealing with FC-type excited states we will find between them the corresponding RES itself which, due to symmetry reasons, is not the ground state in this potential. More precisely, a level with quantum numbers $(n, n)$ corresponds to the $n$th RES (or the ground state, if $n=1)$. Only the ground state generates a potential in which itself is the ground state too. If, e.g., the effective potential is generated by the first RES, the latter having an antisymmetrical wave function, will be the first excited state $\left(n=n^{\prime}=2\right)$ in this potential. It means there exists a ground state with a lower energy (see $\varepsilon_{2,1}$ in Fig. 4) and with a symmetrical wave function. For $n=3$ the corresponding RES is the second FC-type excited state $\left(n=3, n^{\prime}=3\right)$ and there are the ground state $\left(n=3, n^{\prime}=1\right)$ and the first FC-type excited state $\left(n=3, n^{\prime}=2\right)$ with lower energies.

The energies of several states with $n=2$ are shown in Fig. 4 and the contourplots of the wave functions for $n=2, n^{\prime}=1,2$ in Fig. 5. From the ground state energy $\varepsilon_{\text {pol }}=-0.332$ of the $1 \mathrm{D}$ polaron ${ }^{30}$ in a box with length $2 L=7$, and from the value $\varepsilon_{2,1}=-0.679$ in Fig. 4 for the

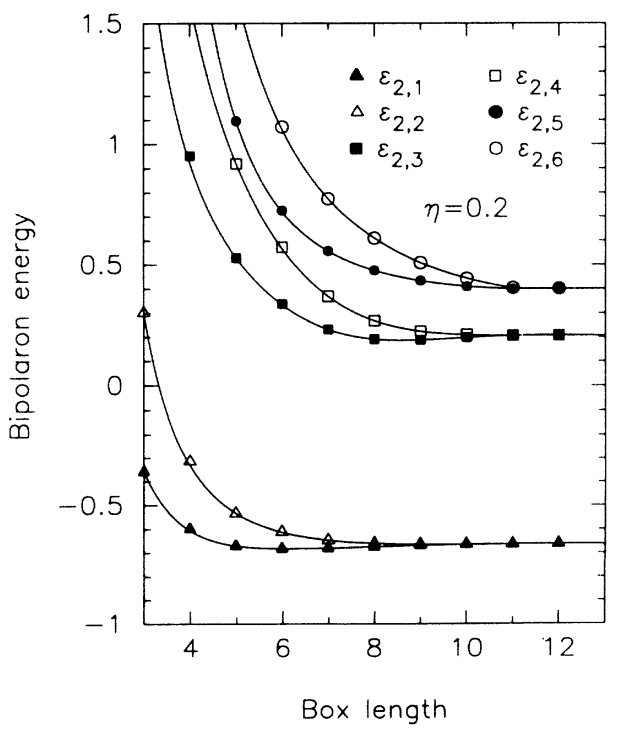

FIG. 4. The energy levels $\varepsilon_{2, n^{\prime}}$ which belong to the states in the potential well generated by the first RES as function of the size of the confinement box (calculated with $N=M=15$ ). 

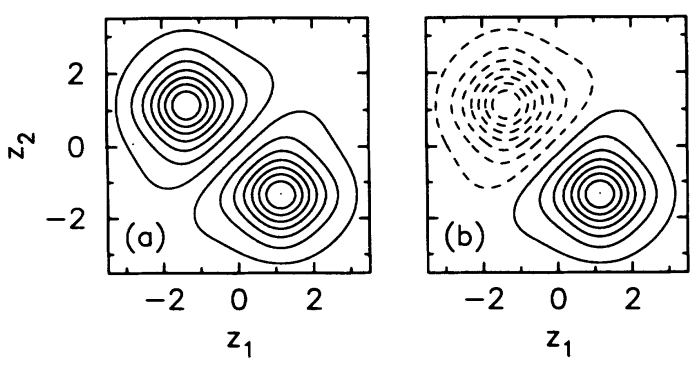

FIG. 5. The contourplots of the wave functions for $n=2$ and $n^{\prime} \leq 2$. The solid line corresponds to positive values of the wave function and the dashed line to negative values: (a) $n^{\prime}=1$, (b) $n^{\prime}=2$.

1D bipolaron in the same box, we can conclude that this ground state in the potential of the first RES is stable and will exist for certain values of the box length $2 L$ and $\eta$ (see also later).

In the limit of an infinitely large box we found that the energies of the excited states are grouped because of symmetry reasons. Let us look at Fig. 6 in which we plot the energy spectrum for $n \leq 3$ and $n^{\prime} \leq n+1(n=3)$ or $n^{\prime} \leq n(n=1,2)$. Apparently, the $\varepsilon_{n, n^{\prime}}$ consist of groups of $n+1$ energy levels if $n=3$, and of $n$ energy levels if $n=2$. The energies $\varepsilon_{3, n^{\prime}}$ for $n^{\prime} \leq 4$ as function of the box length $2 L$ show us that in the limit of an infinitely large box the energy levels indeed combine to groups as discussed above and that in this limit all these energies $\varepsilon_{3, n^{\prime}}\left(n^{\prime} \leq 4\right)$ tend to the energy $\varepsilon_{\mathrm{RES} 2}=\varepsilon_{3,3}$ of the second RES. This energy level is fourfold degenerate. In Fig. 7 we plot the contour plots of those wave functions

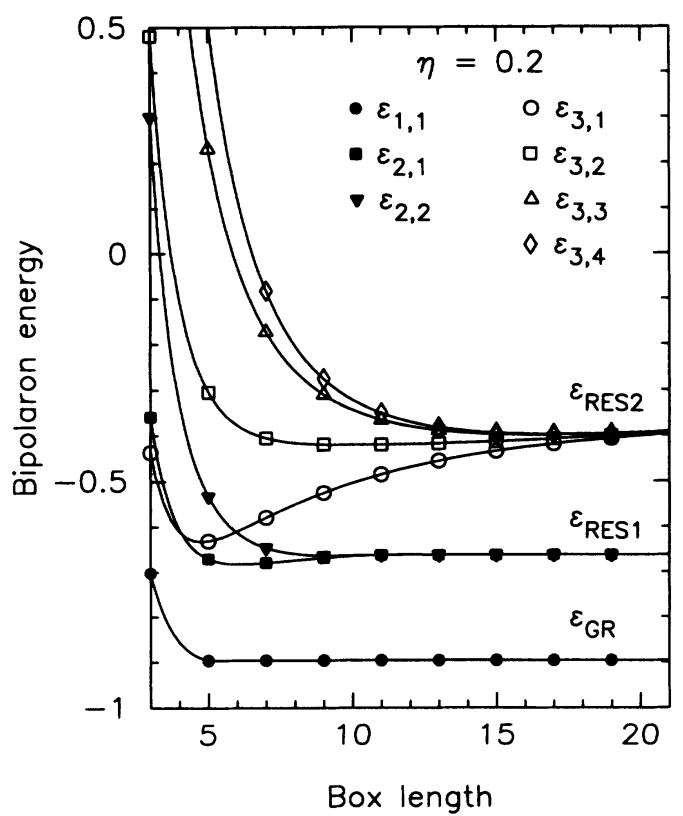

FIG. 6. The energy levels $\varepsilon_{n, n^{\prime}}$ for $n \leq 3$ and $n^{\prime} \leq n+1$ $(n=3)$ or $n^{\prime} \leq n(n=1,2)$. For increasing box length $2 L$ we observe a grouping of energy levels which is a consequence of the symmetry of the wave functions (calculated with $N=M=15$ ).
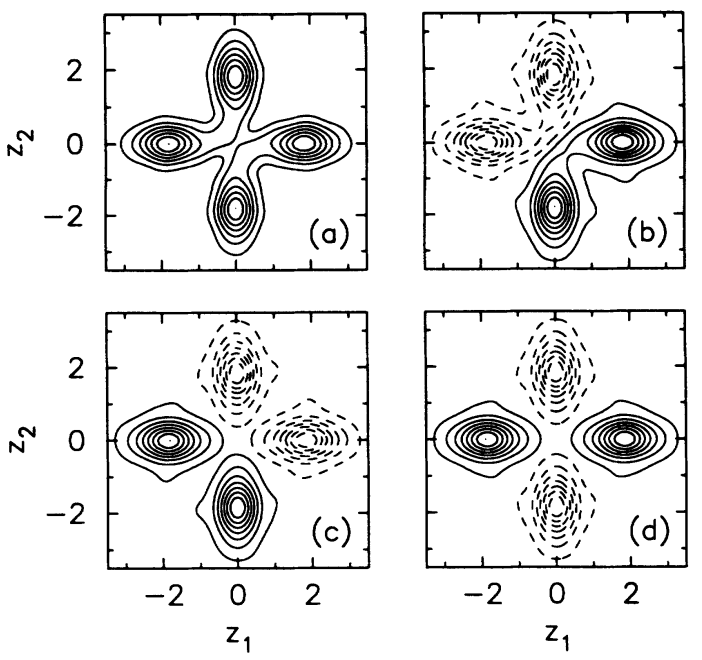

FIG. 7. The contourplots of the wave functions for $n=3$ and $n^{\prime} \leq 4$. The solid line corresponds to positive values of the wave function and the dashed line to negative values: (a) $n^{\prime}=1$, (b) $n^{\prime}=2$, (c) $n^{\prime}=3$, and (d) $n^{\prime}=4$.

for $n=3, n^{\prime} \leq 4$. They correspond to the energies $\varepsilon_{3,1}$ (a), $\varepsilon_{3,2}$ (b), $\varepsilon_{3,3}$ (c), and $\varepsilon_{3,4}$ (d). We see the four symmetries which are allowed by the integro-differential equation (2.6) and cause the degeneracy. For the excited states in the potential generated by the first RES $(n=2)$ the symmetry relations are restricted to two symmetry combinations (see Fig. 5). So we indicated the reasons for the grouping seen in Figs. 4 and 6.

With $\eta=0.2$ the numerical results for the energies of the ground state and the first three RES's in the limit of an infinitely large box and extrapolated to an infinite number of terms in the Fourier series $(N=M \rightarrow \infty)$ are, respectively, $\varepsilon_{1,1}=-0.986, \varepsilon_{2,2}=-0.667, \varepsilon_{3,3}=$ -0.442 , and $\varepsilon_{4,4}=-0.416$. The $\varepsilon_{n, n}$ with $n$ even correspond to an antisymmetrical wave function. In this case the $\delta$ function of the contact potential does not contribute because the wave function is zero at $z_{1}=z_{2}$. However, for the RES states with odd $n$ the $\delta$ function will play an important role.

To understand better the structure of the bipolaron excited states we consider the bipolaron problem with no repulsion $\left(U^{\prime}=0\right)$ between electrons. Then the effective potential becomes separable and bipolaron wave functions can be represented as products of one-polaron wave functions of Ref. 30. Moreover, we consider the box length large enough so that the peaks of the one-particle solutions are infinitely separated.

The results of the study are as follows. First of all there are states which can be represented in this limit as combinations of the same one-particle wave functions or wave functions which have different symmetry properties but with peaks on the same places. Then the resulting bipolaron energy will be 4 times the sum of the one-particle energies due to the strong nonlinear effects in the system (see the Appendix). In particular, the ground state bipolaron wave function at $U^{\prime}=0$ is constructed as 
the product of two one-polaron ground state wave functions and has a peak at $z_{1}=z_{2}=0$. The corresponding energy $A$ equals 4 times the doubled polaron energy: $A=-8 / 3$. When the repulsion is switched on the peak is splitted into two peaks (at a short distance) which are symmetrical to the line $z_{2}=z_{1}$. (cf. Fig. 2). The ground state energy becomes equal to $\varepsilon_{1,1}$. One more example derived from the $U^{\prime}=0$ case is the symmetrized or antisymmetrized product of one-polaron wave functions corresponding to the first RES and to the ground state in the potential generated by this RES. Each one-particle wave function has peaks at the points $z_{i}= \pm L / 2$ and the corresponding one-particle energy is $-1 / 12$ as was found in Ref. 30. The resulting bipolaron wave functions have not four but also two peaks (a positive and a negative one) at the points $z_{2}=z_{1}= \pm L / 2$ for the symmetrical and $z_{2}=-z_{1}= \pm L / 2$ for the antisymmetrical states. At $U^{\prime}=0$ and for an infinite large box these states are degenerate and the corresponding energy is [see Eq. (A11)] $A=4(-2 / 12)=-2 / 3$. When the repulsion is switched on the antisymmetrical state is not influenced [cf. Fig. 5(b)] and the energy corresponds to $\varepsilon_{2,2}$. The peaks of the symmetrical wave function, however, will split [cf. Fig. 7(c)] and its energy will take a higher value depending on $\eta$ which determines the factor in front of the $\delta$-function potential. Its energy for $\eta=0.2$ is equal to $\varepsilon_{3,3}$.

Then there are one-particle states whose wave functions have peaks at different points. The energies of symmetrical and antisymmetrical combinations of such oneparticle states will be the sum of the one-particle energies as it is in linear physics (see the Appendix). From the symmetrical and antisymmetrical combinations of the one-particle ground state and first RES wave functions, we can explain the origin of the third RES $\left(n=n^{\prime}=4\right)$ and obtain exactly the energy $\varepsilon_{4,4}$.

In the same limits as for the above RES states (box length $\rightarrow \infty, N=M \rightarrow \infty$ ) the energies of the ground state, the first RES, and the ground state in the potential of the first RES are shown in Fig. 8 as a function of $\eta$. The $1 \mathrm{D}$ bipolaron ground state is always stable and exists. The first RES, however, has a constant energy $\varepsilon_{\text {RES1 }}=-0.667$ which corresponds to twice the single-polaron energy. This energy is independent of $\eta$ because $\eta$ is only present in the $\delta$-function term which does not contribute to antisymmetrical wave functions. Because of this energy value for the first RES we see that the energy of the ground state (when the box length $\rightarrow \infty, N=M \rightarrow \infty)$ should be lower than this value -0.667. Taking into account that the ground state energy increases with increasing $\eta$ and that the numerically obtained upper bound (within the same limits as above) tends to the same value for $\eta=1.0$, we can conclude that in the limit of an infinite large box $\eta_{c}^{\text {low }} \approx 1$.

In the potential generated by this first RES the ground state has a lower energy $\varepsilon_{2,1}$ which goes asymptotically to $\varepsilon_{\mathrm{RES} 1}$ when $\eta$ approaches unity (infinitely separated electrons). Therefore, this state will be stable except in the case of an infinitely strong repulsion caused by $\eta \rightarrow 1$. Nevertheless, because the energy of the first RES equals twice the single-polaron energy, the lifetime in this first

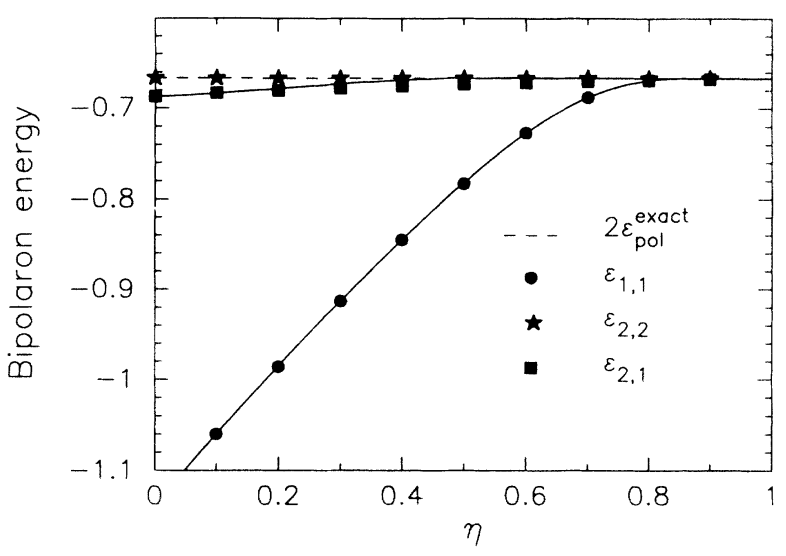

FIG. 8. The energies of the ground state, first RES, and the ground state in the potential generated by this first RES as function of $\eta$ (in the limit $N=M \rightarrow \infty$ ). The curves are guides to the eye.

RES will be very short. To obtain the ground state in the potential generated by the first RES the electrons need to be situated firstly in the first RES itself from where this ground state can be reached. Therefore the probability of finding two electrons in the latter state will be very small despite being stable.

\section{CONCLUSIONS}

The 1D bipolaron ground state has been treated in the limit of strong electron-phonon coupling. We obtained lower bounds for the critical ratio $\eta_{c}$ of the dielectric constants from which we conclude that the stability region for bipolaron formation is considerably larger in 1D as we would obtain in $2 \mathrm{D}$ and $3 \mathrm{D}$. The most important characteristics of the $1 \mathrm{D}$ bipolaron, like the root-meansquared distance between the electrons and the effective bipolaron mass were calculated.

Within the Fourier series approach it is straigthforward to obtain the possible excited states of the bipolaron. We found that in the limit of an infinitely large box the ground state will be bound and the first RES has an energy which equals the energy of two single polarons. The ground state in the potential generated by the first RES is stable. Because of symmetry reasons the excited states combine into groups with the same energy when we consider the limit of an infinitely large box.

\section{ACKNOWLEDGMENTS}

M.A.S. is grateful to the University of Antwerp (UIA) and Eindhoven University of Technology for the support (NWO grant for visitors No. 07-13-137) and the kind hospitality during his visit to the Benelux. F.M.P. is supported by the Belgian National Science Foundation (NFWO). P.V. is supported by a FKFO project, and thanks the NFWO and the Joint Institute for Nuclear 
Research for the support of his visit to Dubna. This work is partly performed in the framework of "Diensten voor de Programmatie van het wetenschapsbeleid" (Belgium) under Contract No. IT/SC/24, the supercomputer project of the NFWO, the FKFO project 2.0093.91, and the ALPHA-project of NFWO-Universiteit Antwerpen.

\section{APPENDIX}

To better understand the structure of the bipolaron excited states let us consider an unphysical situation when $g=0$ that is when the $\delta$-function repulsion potential in Eq. (2.7) is not taken into account. The effective potential becomes separable and a bipolaron wave function can be represented as a symmetrized or an antisymmetrized product of "one-particle" wave functions of Ref. 30:

$$
\chi\left(z_{1}, z_{2}\right)=N\left[\chi_{1}\left(z_{1}\right) \chi_{2}\left(z_{2}\right)+\gamma \chi_{1}\left(z_{2}\right) \chi_{2}\left(z_{1}\right)\right]
$$

where $\gamma=1(-1)$ for symmetrical (antisymmetrical) states and the normalization constant in Eq. (A1) is defined by the relation

$$
1=2 N^{2}\left[1+\gamma\left(\int_{-L}^{L} d z \chi_{1}(z) \chi_{2}(z)\right)^{2}\right]
$$

Inserting the general bipolaron wave function (A1) into Eqs. (2.6), (2.7) we arrive at the equations

$$
\begin{aligned}
\chi_{1}\left(z_{1}\right) & D \chi_{2}\left(z_{2}\right)+\chi_{2}\left(z_{2}\right) D \chi_{1}\left(z_{1}\right) \\
& +\gamma\left[\chi_{1}\left(z_{2}\right) D \chi_{2}\left(z_{1}\right)+\chi_{2}\left(z_{1}\right) D \chi_{1}\left(z_{2}\right)\right]=0
\end{aligned}
$$

where the operator $D$ is defined as follows:

$$
D \chi_{i}(z)=-\frac{1}{2} \chi_{i}^{\prime \prime}(z)+\frac{U_{\infty}-A}{2} \chi_{i}(z)-4 \sqrt{2} N^{2}\left[\chi_{1}^{2}(z)+\chi_{2}^{2}(z)+2 \gamma \chi_{1}(z) \chi_{2}(z) \int_{-L}^{L} d z^{\prime} \chi_{1}\left(z^{\prime}\right) \chi_{2}\left(z^{\prime}\right)\right] \chi_{i}(z)
$$

and the asymptotical value $U_{\infty}$ of the effective potential (2.7) takes the form

$$
\begin{aligned}
U_{\infty}= & 4 \sqrt{2} N^{4}\left\{\int_{-L}^{L} d z\left[\chi_{1}^{2}(z)+\chi_{2}^{2}(z)\right]^{2}+4 \int_{-L}^{L} d z \chi_{1}^{2}(z) \chi_{2}^{2}(z)\left(\int_{-L}^{L} d z \chi_{1}(z) \chi_{2}(z)\right)^{2}\right. \\
& \left.+4 \gamma \int_{-L}^{L} d z \chi_{1}(z) \chi_{2}(z)\left[\chi_{1}^{2}(z)+\chi_{2}^{2}(z)\right] \int_{-L}^{L} d z \chi_{1}(z) \chi_{2}(z)\right\}
\end{aligned}
$$

Solutions to Eq. (A3) can be constructed with eigenvectors of the operator $D$ :

$$
D \chi_{i}(z)=C_{i} \chi_{i}(z)
$$

Inserting into Eq. (A3) we obtain that

$$
C_{1}+C_{2}=0
$$

If the wave functions $\chi_{1}$ and $\chi_{2}$ have different symmetries under reflections, the interception integral

$$
\int_{-L}^{L} d z \chi_{1}(z) \chi_{2}(z)=0
$$

and formulas (A2), (A4), (A5) are simplified. The same occurs in the limit of an infinite large box when $\chi_{1}, \chi_{2}$ have peaks in different places, which are, of course, infinitely separated from each other.

First, we consider bipolaron states which can be constructed from combinations of one-particle wave functions with different symmetry properties but with peaks on the same places. As an example we suppose that $\chi_{1}(z)$ is a symmetrical function in $z$ and $\chi_{2}(z)$ is an antisymmetrical function and suppose also that being squared these functions will coincide in the limit of the infinitely large box. Then we have $N=1 / \sqrt{2}$ and obtain the following equations from Eqs. (A4), (A5):

$$
\begin{aligned}
& -\frac{1}{2} \chi_{1}^{\prime \prime}(z)+\tilde{U}(z) \chi_{1}(z)=\left(\frac{A}{2}+C_{1}\right) \chi_{1}(z), \\
& -\frac{1}{2} \chi_{2}^{\prime \prime}(z)+\tilde{U}(z) \chi_{2}(z)=\left(\frac{A}{2}-C_{1}\right) \chi_{2}(z), \\
& \tilde{U}(z)=2 \sqrt{2} \int_{-L}^{L} d z \chi_{1}^{4}(z)-4 \sqrt{2} \chi_{1}^{2}(z) .
\end{aligned}
$$

After scaling $z_{1(2)} \rightarrow z_{1(2)} / 2$ we can choose as $\chi_{2}$, for example, the first RES of a single polaron, and as $\chi_{1}$ the excited polaron state which is the ground state in the potential generated by $\chi_{2}$. Indeed these functions have the supposed symmetries under reflections and peaks at the points $z= \pm L / 2$ which are infinitely separated in the limit $L \rightarrow \infty$. Thus we obtain from Eq. (A9)

$$
\begin{aligned}
& \frac{A}{8}+\frac{C_{1}}{4}=\varepsilon_{2,1}^{(1)} \\
& \frac{A}{8}-\frac{C_{1}}{4}=\varepsilon_{2,2}^{(1)}
\end{aligned}
$$


where $\varepsilon_{n_{1}, n_{1}^{\prime}}^{(1)}$ is the single-polaron energy in a state with the mentioned quantum numbers of which the first is related to a RES and the second - to an excited state in the potential of that RES. From Eqs. (A7) and (A10) we obtain the expression for the energy of this bipolaron state in the limit of a large box:

$$
A=4\left(\varepsilon_{2,1}^{(1)}+\varepsilon_{2,2}^{(1)}\right)=-\frac{2}{3} .
$$

The general rule is that the energy of a bipolaron state constructed from one-particle wave functions with peaks on the same places equals 4 times the sum of the oneparticle energies. If both particles are in the same state, we arrive at the same general rule (see Ref. 25).

Next, we consider the case when we construct a bipolaron state from wave functions with peaks at different points. In the limit of the infinite length of the box these peaks are infinitely separated. Then we may neglect all products of the type $\chi_{1}^{\nu} \chi_{2}^{\mu}$ in the Eqs. (A4) and (A5). We arrive at the following pair of equations which replaces Eq. (A9):

*Electronic mail address: vansant@nats.uia.ac.be

† Electronic mail address: peeters@nats.uia.ac.be

‡ Permanent address: Bogoliubov Theoretical Laboratory, Joint Institute for Nuclear Research, 141980 Dubna, Russia; electronic mail address: smond@thsun1.jinr.dubna.su

${ }^{\S}$ Permanent address: Universiteit Antwerpen (UIA), Departement Natuurkunde, Universiteitsplein 1, B-2610 Antwerpen, Belgium and also at: Universiteit Antwerpen (RUCA), Groenenborgerlaan 171, 2020 Antwerpen, Belgium; electronic mail address: devreese@nats.uia.ac.be

${ }^{1}$ Polarons and Excitons, edited by C. G. Kuper and G. D. Whitfield (Oliver and Boyd Ltd., Edinburgh, 1963); Polarons in Ionic Crystals and Polar Semiconductors, edited by J. T. Devreese (North-Holland Publishing Company, Amsterdam, 1972); Polarons and Excitons in Polar Semiconductors and Ionic Crystals, edited by J. T. Devreese and F. M. Peeters (Plenum Publishing Corp., New York, 1985).

${ }^{2}$ V. L. Vinetskii, Zh. Eksp. Teor. Fiz. 40, 1459 (1961) [Sov. Phys. JETP 13, 1023 (1961)].

${ }^{3}$ V. K. Mukhomorov, Fiz. Tekh. Poluprovodn. 16, 1095 (1982) [Sov. Phys. Semicond. 16, 700 (1982)].

${ }^{4}$ S. G. Suprun and B. Y. Moizhes, Fiz. Tverd. Tela 24, 903 (1982) [Sov. Phys. Solid State 24, 1153 (1982)].

${ }^{5}$ J. Adamowski, Acta Phys. Pol. A 73, 345 (1988); Phys. Rev. B 39, 3649 (1989).

${ }^{6}$ F. Bassani, M. Geddo, G. Iadonisi, and D. Ninno, Phys. Rev. B 43, 5296 (1991).

${ }^{7}$ G. Verbist, F. M. Peeters, and J. T. Devreese, Phys. Rev. B 43, 2712 (1991).

${ }^{8}$ G. Verbist, M. A. Smondyrev, F. M. Peeters, and J. T. Devreese, Phys. Rev. B 45, 5262 (1992).

${ }^{9}$ G. Verbist, F. M. Peeters, and J. T. Devreese, Solid State Commun. 76, 1005 (1990).

${ }^{10}$ R. Micnas, J. Ranninger, and S. Robaszkiewicz, Rev. Mod. Phys. 62, 43 (1990).

${ }^{11}$ A. S. Alexandrov, JETP Lett. 55, 189 (1992).

${ }^{12}$ M. J. Rosseinsky, A. P. Ramirez, S. H. Glarum, D. W. Murphy, R. C. Haddon, A. F. Hebard, T. T. M. Palstra,

$$
\begin{aligned}
& -\frac{1}{2} \chi_{1}^{\prime \prime}(z)+\breve{U}_{1}(z) \chi_{1}(z)=\left(\frac{A}{2}+C_{1}+\Delta\right) \chi_{1}(z) \\
& -\frac{1}{2} \chi_{2}^{\prime \prime}(z)+\breve{U}_{2}(z) \chi_{2}(z)=\left(\frac{A}{2}-C_{1}-\Delta\right) \chi_{2}(z) \\
& \breve{U}_{i}(z)=\sqrt{2} \int_{-L}^{L} d z \chi_{i}^{4}(z)-2 \sqrt{2} \chi_{i}^{2}(z) \\
& \Delta=\frac{1}{\sqrt{2}} \int_{-L}^{L} d z\left(\chi_{1}^{4}-\chi_{2}^{4}\right)
\end{aligned}
$$

These equations are exactly one-particle equations and so the expression between brackets on the right-hand side is equal to the corresponding one-particle energies. The general rule is now that the energy of a bipolaron state constructed from one-particle wave functions with peaks at different places equals the sum of the one-particle energies, which is usual in linear physics. Considering both general rules a combination of the one-particle ground state and an excited state could lead to a higher bipolaron energy than the combination of two excited states. This peculiarity is caused by the strong nonlinearity of the effective interaction.

A. R. Kortan, S. M. Zahurak, and A. V. Makhija, Phys. Rev. Lett. 66, 2830 (1991).

${ }^{13}$ V. L. Vinetskii and E. A. Pashitskii, Ukr. Fiz. Zh. (Russ. Ed.) 20, 338 (1975) [Ukr. Phys. J. 20, 312 (1975)].

${ }^{14}$ D. Emin, Phys. Rev. Lett. 62, 1544 (1989); D. Emin and M. S. Hillery, Phys. Rev. B 39, 6575 (1989).

${ }^{15}$ K.-F. Berggren, T. J. Thornton, D. J. Newson, and M. Pepper, Phys. Rev. Lett. 57, 1769 (1986).

${ }^{16}$ W. P. Su, J. R. Schrieffer, and A. J. Heeger, Phys. Rev. Lett. 42, 1698 (1979)

${ }^{17}$ M. A. Smondyrev, E. A. Kochetov, G. Verbist, F. M. Peeters, and J. T. Devreese, Europhys. Lett. 19, 519 (1992)

${ }^{18}$ J. T. Devreese, in Polarons in Ionic Crystals and Polar Semiconductors, edited by J. T. Devreese (North-Holland Publishing Company, Amsterdam, 1972), p. 83.

${ }^{19}$ E. Kartheuser, R. Evrard, and J. Devreese, Phys. Rev. Lett. 22, 94 (1969).

${ }^{20}$ J. Devreese, J. De Sitter, and M. Goovaerts, Solid State Commun. 9, 1383 (1971); Phys. Rev. B 5, 2367 (1972).

${ }^{21}$ F. M. Peeters and S. A. Jackson, Phys. Rev. B 31, 7098 (1985); 34, 1539 (1986).

${ }^{22}$ F. M. Peeters, X. Wu, and J. T. Devreese, Phys. Rev. B 33, 3926 (1986).

${ }^{23}$ F. M. Peeters and M. A. Smondyrev, Phys. Rev. B 43, 4920 (1991).

${ }^{24}$ P. Vansant, M. A. Smondyrev, F. M. Peeters, and J. T. Devreese (unpublished).

${ }^{25}$ P. Vansant, F. M. Peeters, M. A. Smondyrev, and J. T. Devreese (unpublished).

${ }^{26}$ E. P. Gross, Ann. Phys. (N.Y.) 99, 1 (1976).

${ }^{27} \mathrm{H}$. Leschke and S. Wonneberger, J. Phys. A 22, L1009 (1989); 23, 1475(E) (1990).

${ }^{28}$ X. Wu, F. M. Peeters, and J. T. Devreese, Phys. Rev. B 31, 3420 (1985).

${ }^{29}$ F. M. Peeters and J. T. Devreese, Phys. Rev. B 36, 4442 (1987).

${ }^{30}$ P. Vansant, M. A. Smondyrev, F. M. Peeters, and J. T. Devreese (unpublished). 

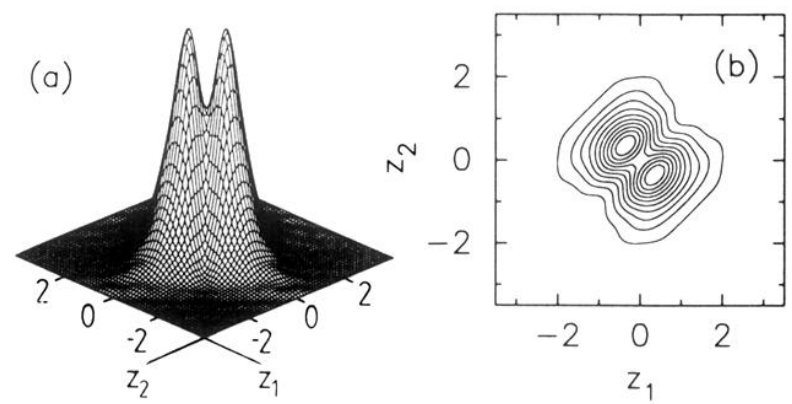

FIG. 2. (a) The 1D bipolaron wave function $\phi\left(z_{1}, z_{2}\right)$ for $\eta=0.2$ as obtained within the Fourier series approach. (b) The contour map corresponding to this bipolaron wave function. 